\title{
The Impact of Tenure Track Personnel System on Higher Education in China
}

\author{
Yihua Wang \\ School of Environment, Education and Development, The University of Manchester, Manchester, M13 9PL, United \\ Kingdom \\ *Corresponding author. Email: gaoming@cas-harbour.org
}

\begin{abstract}
The publications in international journals are widely perceived to be the crucial indicator in global ranking tables. To increase China's competitiveness in the global knowledge economy, the tenure track system is introduced in Chinese elite universities, aiming to realize the transition from key universities from world- class universities. However, the introduction of tenure track system arises some potential influence in practice which is opposite from initial expectation. In this project, the literature review method is used to examine the effectiveness of tenure track system and explore the impacts of tenure track system on higher education. The findings are as follows. Firstly, the tenure track system creates publish-or-perish atmosphere among young faculty members, Under the pressure to publish, young faculty members focus more on the output of academic paper regardless of the quality of academic research. Secondly, as the tenure track system links the promotion opportunity with academic research output, young faculty members are forced to give up the teaching activity, devoting mostly of their time on academic output. Finally, the tenure track system causes administrative work overloads, which breaks the balance between work and personal lives.
\end{abstract}

Keywords: Academic research, Mental Stress, Publish or perish, Teaching assessment, Tenure Track System

\section{INTRODUCTION}

Tenure track personnel system for new junior faculty in China's universities can be seen as a product of globalization. It is well recognized that China realizes and actively engages in the competition with Western hegemony in higher education [1]. As Tian and Lu (2017) point out, after recognizing that the development of higher education is a crucial criterion for developing national competitiveness in the global knowledge economy, China implemented a series of reforms, aiming to improve the quality of the higher education system. One of the measures initiated by the Ministry of Education is to develop its elite academic institutions into world-class universities [2]. As Tian et al. (2016) illustrate, publications are seen as the key indicators of evaluating one university' research strength, closely linked with their rankings in international league tables [1]. To enhance the rankings of China's elite universities in league tables and attract more research funds, China's universities have proposed more stringent requirements on the research productivity of young faculty members. This initiative pushes forward the introduction of Tenure Track Personnel System.

Previous studies mostly defined Tenure Track Personnel System as an incentive and evaluation system. Every three years, promotions and salaries are evaluated against performance metrics defined by each school or department. By the time, teachers who are not eligible for tenure track could choose to leave or remain in the contract system until they are knocked out of the scheme [3]. Tian et al. (2016) adds that a so-called publish-or-perish academic culture has already emerged on campus due to the introduction of tenure track system. The career path of young faculty members is determined by the amount of their publications [1]. Moreover, as Tian and Lu (2017) state, aiming to meet the criterion of assessment, academics have been reported as turning their concentration away from other teaching activities to research outputs which can be measured against internationally recognized standards [2]. As previous literature mostly focuses on student groups to discuss the impact of tenure track system in China, the author will take the perspective of young 
faculty members to examine the effectiveness of tenure track system in China. Besides, the impact of tenure system on academic research; whether this tenure system would arise conflict between teaching and academic research; the impact of tenure track system on faculty members lives will be explored in this paper. By exploring theses three aspects, the author hope can formulate a more clearly understanding regarding to this incentive and evaluation system.

\section{THE IMPACT OF TENURE TRACK PERSONNEL SYSTEM ON ACADEMIC RESEARCH}

The tenure track personnel system would lead to an overemphasis on the productivity of academic research, which can also be seen as the nature of publish-or-perish academic culture. As De Rond and Miller point out, the publish-or-perish signifies that a faculty members' tenure is determined by his or her publications. The reason for publishing articles is obviously for gaining academic respect and tenure, which can also be considered as part and parcel of today's research culture [4]. Lawrence (2008) further adds that the academic publishing system is misinterpreted as a scheme of producing unread, unreadable, and worthless articles used only to advance young faculty members ' academic careers and bloat the libraries [5]. Moreover, Alder and Harzing (2009) hold the view that the tenure track system cannot maximize the productivity of academic research. It is the collaborative atmosphere that inspires and supports the academic research, rather than the publish-or-perish academic culture [6]. Zhang (2014) argues that the tenure track system can be seen as an effective way to help young faculty members achieve academic output. The pressure to publish, especially for tenure purposes, may incent junior faculty members to write articles that would be more easily accepted by journal editors [3]. Zhang' s argument is backed up by Tian et al. (2016), who wrote that the China shows a rapid growth in the number of academic publications in the past decade [1].

Despite the greater research outputs, the tenure track system would negatively effect on the innovation and perceived relevance of academics' research, which would debase the quality of academic research. Other researchers claim that measurement of academic productivity is more difficult than expected [6]. The assessment of tenure track system imposes a flat, acrossthe -board rule. As Tian and Lu (2017) point out, the research in social sciences and humanities $(\mathrm{SSH})$ is placed at a disadvantage position compared to science and technology(S\&T) disciplines. The reason behind this phenomenon is that academic researchers in social sciences disciplines would focus more on seeking different ideologies and discourses [2]. One-size-fits-all approach in tenure track system would force young faculty members to downgrade their research aims, making transition from making discoveries to publishing as many academic journal articles as possible [6]. Thus, the tenure track system would damage the practice of academic research as the motivation of researchers is transformed. The accuracy and objectivity deteriorate [5]. Moreover, a recurring concern on the tenure track system is its emphasis on the number of publications. Any progress in academic output is perceived to come at the expense of creativity and innovation [4]. This argument is supported by Tian and Lu (2017), who write that the academic researchers are more likely to give up the academically significant research topics that take longer time to explore. To meet the tenure requirements on academic output, young faculty members would prefer to follow the existing research topic and research methods [2]. Tian and Lu' s concern is in line with other researchers' criticism of tenure track system. Lawrence (2008) holds the view that tenure contracts prevent young faulty members from working on permanent academic research and fulfilling their academic research. The quantitative evaluation of publications (i.e. calculating the number of items) affect the quality of their research [5]. De Rond and Miller (2005) also claim greater academic output is perceived as relatively incremental, lacking in significance and substance, and overly repeating the obvious. The reason why tenure track system inhibits young faculty members , innovation is that their findings could irritate deeply entrenched preconceptions in a specific profession [4]. Although the tenure track system would promote the output of internationally indexed publications [1], it is well recognized that the tenure track personnel system threatens and suppresses the academic and moral values traditionally embedded in higher education. The academic freedom and equalities can be marginalized and jeopardized [2]. As De Rond and Miller (2015) Illustrate, authors who often refuse to accept revision proposals from editors and reviewers are less likely to publish enough articles in refereed journals to survive in tenure track system [4]. Furthermore, Tian et al. (2016) hold the view that over-stressing the number of publications in the tenure track system is to blame for the rise in research misconduct. They further add that the tenure track system has changed the function of publications, journal articles are used for getting promotion and chasing academic refunds instead of sharing ideas among academic community. The publications not only bring the recognition and honor to young faculty members, but also potentially encourage them to take risks of publishing fraudulent papers [1]. 


\section{THE IMPACT OF TENURE TRACK PERSONNEL SYSTEM ON TEACHING ACTIVITY}

The rigid tenure track personnel system requirement not only debases the quality of research, but also reduces the efforts that young faculty members could have put into teaching. For instance, today's young faculty members are expected to publish innovative articles, while providing high-quality teaching for large classes [7]. The tenure track personnel system is widely perceived to have a negative effect on teaching activity. As Tian and $\mathrm{Lu}$ (2017) illustrate, lecturers who sign the tenure contracts in science and engineering will no longer need to do teaching. And in all disciplines of the humanities and social sciences, the teaching requirements for lecturers are much lower after implementing tenure track personnel system [2]. This is supported by Zhang (2009), who claims that tenure track system kicks out the teaching from central position [8]. It is well recognized that research and teaching are not compatible. They compete in terms of time [2]. Thus, young faculty members place a high value on research and regard teaching as a low-status occupation. Teaching quality is negatively influenced and the will to innovate in the classroom is hindered. However, this measurement attracts other researchers' criticism. Ylijoki and Ursin (2013) point out that the most obvious problem of the Tenure-Track system and its underlying management approach is to put academic pressure on young faculty members [9]. Putting academic research ahead of teaching and related teaching activities has influenced the teaching of young faculty members and their participation in the growth of their profession as teacher educators. Further concern here is that the degree of marginalization of teaching may in turn negatively affect the academic development of university students [10]. This is because that young faculty members are pushed to achieve short-term goals at the expense of long-term goals and to narrow their career development simply for publishing articles [1]. De Rond and Miller (2005) argues that adamic research serves the purpose in making record on the intellectual history [4]. This is objected by Zhang (2009), holding the view that the tenure track personnel system is based on the quality of personnel training, seen as the value of education [8]. Therefore, the teaching activity is the core and essence of tenure track system.

\section{THE IMPACT OF TENURE TRACK PERSONNEL SYSTEM ON UNIVERSITY TEACHERS' PRESSURES}

The transition to higher education caused by the tenure track system has created a huge workload for faculty members in China' $s$ universities, which requires them to acquire more skills and knowledge to carry out their work. In addition, the lack of research funding is believed to exert another type of pressure on university teachers. As Li and Kou (2018) illustrate, teachers are facing a rapidly changing social environment, increasing information, high speed of life, and factors affecting the workload, which impose excessive demands on work [10]. Li and Kou' $\mathrm{s}$ statement is approved by Tian and Lu (2017), who claims that higher education has evolved from a collegial setting to one that is more market oriented. In the new market model, the demand for academic works has increased dramatically without sufficient compensation, which turn universities into relatively stressful workplaces [2]. Similarly, Tian et al. (2016) agree that China has considerably expanded research expenditure in the last decade, recognizing research productivity as critical to economic competitiveness. To compete for government funding, Chinese universities are putting a lot of pressure on its research-active faculty to publish in journals that appear in the Science Citation Index (SCI) and the Social Science Citation Index (SSCI) [1]. Adler and harzing (2009) also question whether the current tenure track system used to rank young faculty members put much more pressure on scholars, rather than rewarding them [6]. Although some faculty members may experience greater psychological arousal, which can help improve the effectiveness of their work performance further [7], the overtime work have reduced their engagement in family life [2]. Moreover, the tenure track personnel system can reinforce the effects on gender inequalities. As previous literatures indicate, family awareness of gender roles is a main barrier to women's entrepreneurship. Women bear a disproportionate share of family responsibilities and childcare burdens compared to men [11]. Tian and $\mathrm{Lu}$ (2017) also state that female faculty members who are more likely to work in an external context of increased family duties, are disproportionately disadvantaged by the managerial change. As a result, hegemonic male domination in higher education is strengthened [2]. What is more, researchers find that female professors are under more pressure to publish in peer-reviewed journals, and are less satisfied with the publishing process than their male counterparts [12].

\section{CONCLUSION}

To sum up, under the tenure track personnel system, young faculty members face much more pressure than before. Publications are seen as the main goal as it is perceived to be the young faculty members' lifeline. The tenure track system has both positive and negative influences on Chinese universities, teachers. Positively, young faculty members regard the tenure track personnel system as a good way to get high rewards and opportunity for upward mobility. Moreover, they regard the requirement as an objective assessment of their research performance, and they are 
committed to writing academic literature for publication. Negatively, young faculty members express concern about work pressure. With the aim to meet tenure requirements, they feel they do nor have enough time to produce higher- quality academic papers which can have a greater impact on their own professions. Similarly, aiming to increase productivity, young faculty members sacrifice much more time which are supposed to put on teaching activities. Finally, it describes the fact that academic works are increasingly subject to inevitable administrative responsibilities, which are usually assigned from the top and have no real possibility of affecting them. This is seen as a threat to the nature of academia, as an uncontrollable and convincing force to transform the nature of academic work and identity. As previous literature suggests that female faculty members report being more discouraged than their male counterparts when it comes to pursuing creative or non-traditional research. In the further study, the potential impact of tenure track personnel system on females should be focused on to find whether there exists gender bias on academic research and promotion.

\section{REFERENCES}

[1] Tian, M., Su, Y., \& Ru, X. Perish or publish in China: Pressures on young Chinese scholars to publish in internationally indexed journals. Publications, 2016, 4(2), 9.

[2] Tian, M., \& Lu, G. What price the building of world-class universities? Academic pressure faced by young lecturers at a research-centered University in China. Teaching in Higher Education, 2017, 22(8), 957-974.

[3] Zhang, J. Developing excellence: Chinese university reform in three steps. Nature News, 2014, 514(7522), 295.

[4] De Rond, M., \& Miller, A. N. Publish or perish: bane or boon of academic life?. Journal of management inquiry, 2005, 14(4), 321-329.

[5] Lawrence, P. A. Lost in publication: how measurement harms science. Ethics in science and environmental politics, 2008, 8(1), 9-11.

[6] Adler, N. J., \& Harzing, A. W. When knowledge wins: Transcending the sense and nonsense of academic rankings. Academy of Management Learning \& Education, 2009, 8(1), 72-95.

[7] Zábrodská, K., Mudrák, J., Šolcová, I., Květon, P., Blatný, M., \& Machovcová, K. Burnout among university faculty: The central role of work-family conflict. Educational Psychology, 2018, 38(6), 800-819.
[8] Jianlin, Z. Relation between Teaching and Quality: Assumption Underlying the First Round of Undergraduate Teaching Assessment in China. Modern University Education, 2009, 03.

[9] Ylijoki, O. H., \& Ursin, J. The construction of academic identity in the changes of Finnish higher education. Studies in Higher Education, 2013, 38(8), 1135-1149.

[10] Li, W., \& Kou, C. Prevalence and correlates of psychological stress among teachers at a national key comprehensive university in China. International journal of occupational and environmental health, 2018, 24(1-2), 7-16.

[11] Wang, Q., \& Lin, M. Work-family policy and female entrepreneurship: Evidence from China's subsidized child care program. China Economic Review, 2019, 54(C), 256-270.

[12] Miller, A. N., Taylor, S. G., \& Bedeian, A. G. Publish or perish: Academic life as management faculty live it. Career development international. 2011. 